\section{Perspectives}

Histone H1.2 joins an ever-increasing list of proteins that undergo nucleocytoplasmic shuttling prior to or during apoptosis. The key question for each and every such protein is whether the observed shuttling is a cause or a consequence of the apoptotic program. A conclusive answer to this question may not be easy, as studying a dying cell poses a fundamental challenge in differentiating causes and effects. The case for histone H1.2 appears to be strong, as the authors employed a plethora of techniques and assays, including using recombinant $\mathrm{H} 1.2$ to induce cytochrome $c$ release in vitro. Nevertheless, the mechanism underlying the activity of histone H1.2 remains completely unknown. Elucidating the molecular mechanism of mitochondrial protein release is the central yet daunting task facing today's apoptosis researchers. 1. Fernandez-Flores, A., Aguilera, B., Yau, P. \& Oliva, H.
Lancet 359, 1072 (2002).
2. Kerr, J.F.F., Wyllie, A.H. \& Currie, A.R. Br. J. Cancer
26, 239-257 (1972).
3. Wang, X. Genes Dev. 15, 2922-2933 (2001).
4. Konishi, A. et al. Cell 114, 673-688 (2003).
5. Cory, S. \& Adams, J.M. Nat. Rev. Cancer2, 647-656 (2002).

6. Scorrano, L. \& Korsmeyer, S.J. Biochem. Biophys. Res. Commun. 304, 437-444 (2003).

7. Shi, Y. Structure 10, 285-288 (2002).

8. Shi, Y. Mol. Cell 9, 459-470 (2002).

9. Thornberry, N.A. \& Lazebnik, Y. Science 281, 1312-1316 (1998).

10. Li, P. et al. Cell 91, 479-489 (1997).

11. Du, C., Fang, M., Li, Y. \& Wang, X. Cell 102, 33-42 (2000).

12. Verhagen, A.M. et al. Cell 102, 43-53 (2000).

13. Chai, J. et al. Nature 406, 855-862 (2000).

14. Li, W. et al. Nat. Struct. Biol. 9, 436-441 (2002).

15. Jones, J.M. et al. Nature 425, 721-727 (2003).

16. Wei, M.C. et al. Science 292, 727-730 (2001).

17. Puthalakath, H. \& Strasser, A. Cell Death Differ. 9 , 505-512 (2002).

18. Li, H. et al. Science 289, 1159-1164 (2000).

19. Marchenko, N.D., Zaika, A. \& Moll, U.M. J. Biol. Chem. 275, 16202-16212 (2000).

\title{
Telomere tail in a Pot
}

Telomeres consist of tandem repeats of short DNA sequences at the end of linear eukaryotic chromosomes. A number of proteins have been found to bind the telomere DNA and protect its free end from degradation, from undergoing fusion with other telomeres or broken chromosomes, or from being recognized as a broken chromosome. In many species, a 3' single-stranded tail further extends from the duplex telomeric repeat DNA, and Pot1 (protection of telomeres), a protein that is essential for maintaining telomere length, interacts specifically with the telomeric ssDNA.

A recent paper by Lei et al. (Nature, in the press) has demonstrated how fission yeast Pot1 protein recognizes the telomeric overhang. These authors crystallized the $\mathrm{N}$-terminal DNA-binding domain of Pot1 in the presence of telomeric repeat oligonucleotides (5'-GGTTAC-3'). As predicted from sequence analysis, this region forms a classic oligonucleotide-binding (OB) fold (blue space-filling model) characterized by a groove in which the nucleic acid lies. An additional feature to the OB fold in Pot 1 is the presence of two loops (yellow), which line the groove and contact opposite faces of the ssDNA.

Although the structure of the Pot 1 nucleic acid-binding domain offers few surprises, the telomeric ssDNA bound in this domain has many unusual features. The ssDNA (red) adopts a folded, compact structure involving two types of interactions. First, the three sets of neighboring bases (G1-G2; T3-T4; A5-C6) undergo stacking; this involves contacts with hydrophobic and aromatic residues of Pot 1. Second, and even more interestingly, two $G \bullet T$ base pairs are formed: G1•T3 and G2•T4. The latter of these contributes more strongly to the formation of the compact DNA structure. Nevertheless, substitution of either $\mathrm{T}$ with a $\mathrm{C}$ abolishes DNA binding, demonstrating the importance of the compacted structure for stable interaction with

Pot1. These unique base pair interactions fold the DNA backbone nearly perpendicular to the stacking interactions. Although previous results suggested that Pot1 might facilitate telomere extension by telomerase, the 3' end of the oligonucleotide remains buried within the protein cavity in the structure. This raises the question of how this end might become accessible to telomerase activity.

Unlike Pot 1, some OB fold-containing proteins (such as BRCA2 and RPA) bind nucleic acid independently of sequence. Lei et al. compared the OB folds of sequence-specific and non-specific binding proteins and observed that while base-stacking interactions are common to both types of proteins, other interactions such as the unique self-recognition described above, or a second OB fold, are necessary to confer the ability to recognize a specific sequence. The structural study therefore also highlights how, even within the context of a wellestablished structural domain, subtle changes can impart additional specificity that make a protein's function unique.

Angela K Eggleston 